\title{
Vorbemerkung zur 7. Auflage
}

Wegen der regen Nachfrage war die 6. Auflage relativ schnell vergriffen. In der vorliegenden 7. Auflage wurden alle Kapitel überarbeitet und ergänzt.

Der Teil Bakteriologie, Virologie wurde von Herrn Professor Carlson neu verfaßt insbesondere werden die neue Trinkwasserverordnung* und die neuen Bestimmungen über Mineral- und Heilwasser berücksichtigt.

Der Teil Abwasser wurde von Herrn Professor Rüffer auf den neuesten Stand gebracht. So muß es dankbar begrüßt werden, daß beide Mitautoren wieder bereit waren, an dieser Neuauflage mitzuarbeiten.

Hannover, Januar 1986

Karl Höll

* Die Trinkwasserversorgung für die Bevölkerung erfolgt durch eine Vielzahl großer und kleiner Wasserversorgungsanlagen. Hydrogeologische Gegebenheiten, unterschiedliche Bodennutzung und Umweltbelastung bestimmen die Beschaffenheit des Wassers. In einigen Gebieten läßt sich eine Nutzung von Oberflächenwasser nicht vermeiden, in anderen stehen Brunnen mit guter Wasserqualität zur Verfügung. Um die Sicherheit und Akzeptans des Trinkwassers und des Wassers für Lebensmittelbetriebe zu gewährleisten, ist eine gute Technik in den Wasserversorgungsunternehmen und ein gutes Úberwachungssystem notwendig.

Die Anforderungen an die Beschaffenheit des Trinkwassers, des Wassers für Lebensmittelbetriebe sowie die Uberwachung der Wassergewinnungs- und Wasserversorgungsanlagen sind in der Verordnung über Trinkwasser und über Wasser für Lebensmittelbetriebe (Trinkwasser-Verordnung, TrinkwV) festgelegt. Die gesetzlichen Grundlagen für diese Verordnung bilden das Bundesseuchengesetz und das Lebensmittel- und Bedarfsgegenständegesetz.

Die TrinkwV vom 31. Januar 1975, zuletzt geändert durch die Verordnung vom 1. August 1984, ist zur Umsetzung der Richtlinie des Rates der Europäischen Gemeinschaft vom 15. Juli 1980 über die Qualität von Wasser für den menschlichen Gebrauch neu gefaßt worden. Die Anforderungen an die bakteriologischen, physikalischen, physikalisch-chemischen und chemischen Untersuchungen und die Wertung ihrer Ergebnisse wurden dem Stand der wissenschaftlichen Erkenntnis angepaßt.

Um den Hygienestandard zu gewährleisten, müssen die mikrobiologischen Anforderungen dauernd erfüllt sein. Die in der Verordnung festgesetzten Werte für Stoffe und Kenngrößen orientieren sich an den Werten der Richtlinie des Rates. Bei ihrer Einhaltung ist nach dem gegenwärtigen Stand der wissenschaftlichen Erkenntnis ein lebenslanger Gebrauch des Trinkwassers unbedenklich. 